\title{
Addendum to: Solar neutrino oscillation parameters after first KamLAND results
}

\author{
G.L. Fogli, ${ }^{1}$ E. Lisi, ${ }^{1}$ A. Marrone,${ }^{1}$ D. Montanino, ${ }^{2}$ A. Palazzo,${ }^{1}$ and A.M. Rotunno ${ }^{1}$ \\ 1 Dipartimento di Fisica and Sezione INFN di Bari \\ Via Amendola 173, 70126 Bari, Italy \\ ${ }^{2}$ Dipartimento di Scienza dei Materiali and Sezione INFN di Lecce \\ Via Arnesano, 73100 Lecce, Italy
}

\begin{abstract}
In a previous paper [1], we presented a three-flavour oscillation analysis of the solar neutrino measurements and of the first data from the KamLAND experiment, in terms of the relevant massmixing parameters $\left(\delta m^{2}, \theta_{12}, \theta_{13}\right)$. The analysis, performed by including the terrestrial neutrino constraints coming from the CHOOZ (reactor), KEK-to-Kamioka (K2K, accelerator) and SuperKamiokande (SK, atmospheric) experiments, provided a stringent upper limit on $\theta_{13}$, namely, $\sin ^{2} \theta_{13}<0.05$ at $3 \sigma$. We reexamine such upper bound in the light of a recent (although preliminary) reanalysis of atmospheric neutrino data performed by the SK collaboration, which seems to shift the preferred value of the largest neutrino square mass difference $\Delta m^{2}$ downwards. By taking the results of the SK official reanalysis at face value, and by repeating the analysis in [1] with such a new input, we find that the upper bound on $\theta_{13}$ is somewhat relaxed: $\sin ^{2} \theta_{13}<0.067$ at $3 \sigma$. Related phenomenological issues are briefly discussed.
\end{abstract}

PACS numbers: 26.65.+t, 13.15.+g, 14.60.Pq, 91.35.-x 
In a previous paper [1], we presented a three-flavor oscillation analysis of data from KamLAND [2] and solar [3] neutrino experiments, in terms of the three mass-mixing parameters relevant for this data set, namely, the smallest square mass difference $\left(\delta m^{2}=m_{2}^{2}-m_{1}^{2}\right)$, and the two mixing angles between the first and the other two neutrino generations $\left(\theta_{12}\right.$ and $\theta_{13}$ in standard notation [4]). The analysis included also the constraints coming from the following terrestrial neutrino experiments: CHOOZ (reactor) [5], KEK-to-Kamioka (K2K, accelerator) [6], and Super-Kamiokande (SK, atmospheric) [7]. In particular, an approximate $\mathrm{SK}+\mathrm{K} 2 \mathrm{~K}$ data combination [8] was used to constrain the largest neutrino square mass difference: $\Delta m^{2}=(2.7 \pm 0.4) \times 10^{-3} \mathrm{eV}^{2}(1 \sigma)[8]$. The parameter $\Delta m^{2}$ was then marginalized away in the $3 \nu$ analysis of the $\mathrm{CHOOZ}$ spectral data 1, 8], which depends on the four parameters $\left(\Delta m^{2}, \delta m^{2}, \sin ^{2} \theta_{12}, \sin ^{2} \theta_{13}\right)$ 9].

Our summary of the constraints on the $3 \nu$ parameters $\left(\delta m^{2}, \sin ^{2} \theta_{12}, \sin ^{2} \theta_{23}\right)$ was given in Fig. 9 of [1], in terms of the projected $\Delta \chi^{2}$ functions of the global (solar+terrestrial) data fit. The same functions are reported, for the sake of completeness, in Fig. 1 (solid curves). In particular, the following upper bound was obtained on the mixing angle $\theta_{13}[1]$ ):

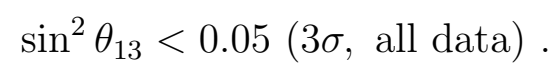

After the work [1], a thorough analysis [10] of the first K2K spectral data [6] has provided, in combination with SK atmospheric neutrino data 7], a more reliable and accurate estimate of the $\Delta m^{2}$ range [10]:

$$
\Delta m^{2}=(2.6 \pm 0.4) \times 10^{-3} \mathrm{eV}^{2}(1 \sigma, \mathrm{SK}+\mathrm{K} 2 \mathrm{~K}) .
$$

A very similar range has been found in a recent, independent analysis of SK $+\mathrm{K} 2 \mathrm{~K}$ data 11 . However, the mere decrease of the $\Delta m^{2}$ best-fit value from 2.7 [8] to 2.6 [10, 11] (in units of $10^{-3} \mathrm{eV}^{2}$ ) does not induce any perceptible change in the results summarized in Fig. 9 of 1]. A nonnegligible change can be instead induced by a more substantial decrease of $\Delta m^{2}$, as possibly indicated by a new SK data reanalysis [12].

The SK Collaboration has recently presented the preliminary results of a global reanalysis of the previous atmospheric neutrino data (no new data included), which incorporates improvements or changes of various basic ingredients, such as the neutrino interaction simulator, the inner and outer detector simulators, the data reduction process, the event reconstruction algorithm, and the input atmospheric neutrino fluxes [12]. It is claimed that each change slightly shifts the $\Delta m^{2}$ allowed region to lower values, the final best-fit value being $\Delta m^{2}=2 \times 10^{-3} \mathrm{eV}^{2}[12$, i.e., $1.5 \sigma$ below the central value in Eq. (2).

At present, we cannot recover from [12] enough information to implement the above variations in a thorough, ab initio analysis of the SK atmospheric data, and thus we cannot independently check the above claim. However, it is tempting to study, at least in a first approximation, the implications of relatively low $\Delta m^{2}$ values [12] on our previous $3 \nu$ analysis 1]. In particular, it is immediate to recognize [13] that a significant downward shift of $\Delta m^{2}$ weakens the upper bound on $\sin ^{2} \theta_{13}$ coming from the CHOOZ data [5]. Since the parameter $\sin ^{2} \theta_{13}$ has an enormous impact on basically all aspects of current and future neutrino phenomenology, we think it useful to reexamine its previous probability distribution [1] in the light of the preliminary SK revised results [12].

To this purpose, we derive an approximate $\chi_{\mathrm{SK}^{\prime}}^{2}\left(\Delta m^{2}\right)$ function through graphical reduction of the SK mass-mixing parameter fit in [12], and combine it with our (unaltered) $\chi_{\mathrm{K} 2 \mathrm{~K}}^{2}\left(\Delta m^{2}\right)$ function from the $\mathrm{K} 2 \mathrm{~K}$ spectral analysis in [10]. We obtain then the following 
"revised" estimate for $\Delta m^{2}$,

$$
\Delta m^{2}=\left(2.0_{-0.3}^{+0.4}\right) \times 10^{-3} \mathrm{eV}^{2}\left(1 \sigma, \mathrm{SK}^{\prime}+\mathrm{K} 2 \mathrm{~K}\right),
$$

where the errors, although asymmetric, turn out to scale linearly up to $\sim 3 \sigma .{ }^{1}$ Notice that the above estimate is compatible with the $\Delta m^{2}$ ranges independently preferred by the final analyses of the MACRO [14] and Soudan 2 [15] atmospheric neutrino experiments.

Assuming the $\Delta m^{2}$ input from Eq. (3), we proceed to perform the global $3 \nu$ fit as in 1], all other phenomenological inputs being unchanged. The results are shown in Fig. 1 (dotted curves). As compared with the previous results [1] (solid curves), no noticeable change is seen in the fit to the leading solar $\nu$ parameters $\delta m^{2}$ and $\sin ^{2} \theta_{12}$ (left and middle panel in Fig. 1). The constraints on $\sin ^{2} \theta_{13}$ are instead somewhat relaxed, as expected. In particular, the intercept of the line at $\Delta \chi^{2}=9$ with the dotted curve in the right panel of Fig. 1 provides the "revised" $3 \sigma$ upper bound on $\sin ^{2} \theta_{13}$,

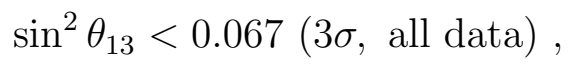

to be compared with the previous one in Eq. (11). Essentially, the main effect of the "revised" SK atmospheric neutrino results [12] consists in enlarging the upper bounds on $\sin ^{2} \theta_{13}$ by a factor $\sim 1.3$ at any C.L. This is the main result of our Addendum.

We conclude with a few qualitative comments on possible phenomenological implications of the relatively "low" $\Delta m^{2}$ in Eq. (3) and of the relatively "weak" upper bound on $\sin ^{2} \theta_{13}$ in Eq. (44). Concerning long-baseline accelerator experiment, an increase in the upper limit on $\sin ^{2} \theta_{13}$ may, in general, enlarge the discovery potential in the $\nu_{\mu} \rightarrow \nu_{e}$ channel. On the other hand, low values of $\Delta m^{2}$ imply small $\nu_{\mu} \rightarrow \nu_{\tau}$ appearance rates $\left[\propto\left(\Delta m^{2}\right)^{2}\right]$ in the same class of experiments. Weakening the bounds on $\sin ^{2} \theta_{13}$ might also weaken future KamLAND limits on $\theta_{12}$ (when they will become competitive with solar neutrino limits), since variations of $\sin ^{2} \theta_{12}$ can be partly traded for variations of $\sin ^{2} \theta_{13}$ (both affecting the KamLAND event rate in a similar way). Uncertainties on the mixing angles may then affect other observables, e.g., the effective Majorana mass in neutrinoless double beta decay. Concerning atmospheric neutrinos, low values of $\Delta m^{2}$ and relatively high values of $\sin ^{2} \theta_{13}$ can make subleading $3 \nu$ effects somewhat more important. In particular, if the best-fit value of $\delta m^{2}$ in Fig. 1 would increase with future KamLAND data, then atmospheric neutrino analyses at zeroth order in $\delta m^{2} / \Delta m^{2}$ might need an upgrade to include higher-order effects. Of course, none of the above effects can be large enough to change significantly the overall picture of the $3 \nu$ oscillation phenomenology. However, current or predicted ranges for several parameters and observables might need small readjustments, should the revised SK atmospheric neutrino analysis in 12] and the $\Delta m^{2}$ estimate in Eq. (3) be basically confirmed by more detailed studies: The "revised" upper limit on $\sin ^{2} \theta_{13}$ in Eq. (4) is just one relevant example.

\section{Acknowledgments}

This work was in part supported by the Italian Ministero dell'Istruzione, Università e Ricerca (MIUR) and Istituto Nazionale di Fisica Nucleare (INFN) within the "Astroparticle

\footnotetext{
${ }^{1}$ In other words, the function $\chi_{\mathrm{SK}^{\prime}+\mathrm{K} 2 \mathrm{~K}}^{2}$ is well approximated by two half-parabolae. The SK' label indicates that we are using here the "revised" Super-Kamiokande results from 12].
} 
Physics" research project.

[1] G.L. Fogli, E. Lisi, A. Marrone, D. Montanino, A. Palazzo and A.M. Rotunno, "Solar neutrino oscillation parameters after first KamLAND results," Phys. Rev. D 67, 073002 (2003).

[2] KamLAND Collaboration, K. Eguchi et al., Phys. Rev. Lett. 90, 021802 (2003).

[3] Homestake Collaboration, B.T. Cleveland, T. Daily, R. Davis Jr., J.R. Distel, K. Lande, C.K. Lee, P.S. Wildenhain, and J. Ullman, Astrophys. J. 496, 505 (1998); SAGE Collaboration, J.N. Abdurashitov et al., J. Exp. Theor. Phys. 95, 181 (2002) [Zh. Eksp. Teor. Fiz. 95, 211 (2002)]; GNO Collaboration, T.A. Kirsten em et al., in the Proceedings of Neutrino 2002, 20th International Conference on Neutrino Physics and Astrophysics (Munich, Germany, 2002), edited by F. von Feilitzsch and N. Schmitz, Nucl. Phys. B Proc. Suppl. 118, 33 (2003); Super-Kamiokande Collaboration, S. Fukuda et al., Phys. Lett. B 539, 179 (2002); SNO Collaboration, Q.R. Ahmad et al., Phys. Rev. Lett. 89, 011302 (2002). Solar neutrino fluxes from: J.N. Bahcall, M.H. Pinsonneault, and S. Basu, Astrophys. J. 555, 990 (2001).

[4] Review of Particle Physics, K. Hagiwara et al., Phys. Rev. D 66, 010001 (2002).

[5] CHOOZ Collaboration, M. Apollonio et al. Phys. Lett. B 466, 415 (1999); Eur. Phys. J. C 27, 331 (2003).

[6] K2K Collaboration, M.H. Ahn et al., Phys. Rev. Lett. 90, 041801 (2003).

[7] E.T. Kearns, "Atmospheric neutrinos in 2002," in HQEL 2002, Proceedings of the 6th International Workshop on Heavy Quarks and Leptons (Vietri sul Mare, Salerno, Italy, 2002), Frascati Phys. Ser. 28, 413 (2002) hep-ex/0210019.

[8] G.L. Fogli, G. Lettera, E. Lisi, A. Marrone, A. Palazzo, and A.M. Rotunno, Phys. Rev. D 66, 093008 (2002).

[9] G.L. Fogli, E. Lisi, and A. Palazzo, Phys. Rev. D 65, 073019 (2002).

[10] G.L. Fogli, E. Lisi, A. Marrone and D. Montanino, Phys. Rev. D 67, 093006 (2003).

[11] M. C. Gonzalez-Garcia and C. Pena-Garay, hep-ph/0306001.

[12] Y. Hayato, "Status of the Super-Kamiokande, the K2K and the J-PARC $\nu$ project," talk at HEP 2003, International Europhysics Conference on High Energy Physics (Aachen, Germany, 2003). Website: eps2003.physik.rwth-aachen.de .

[13] July 2003 Long Baseline Neutrino Oscillation Newsletter (by M.C. Goodman), available at www.hep.anl.gov/ndk/longbnews .

[14] G. Giacomelli, "Final results of the MACRO experiment on atmospheric neutrino oscillations," talk at HEP 2003 [12].

[15] Soudan 2 Collaboration, M. Sanchez et al., hep-ex/0307069 


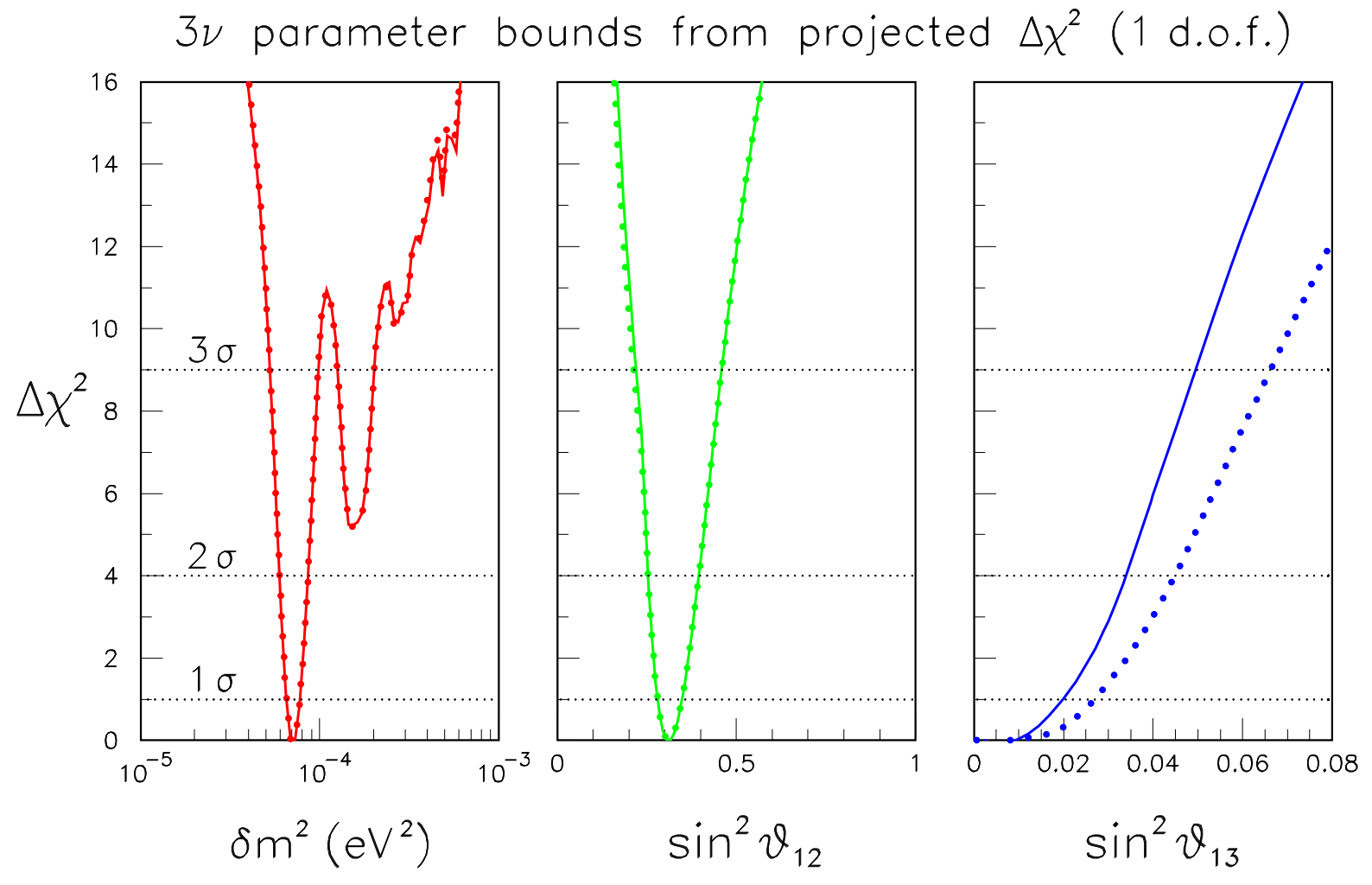

FIG. 1: Three-flavor neutrino oscillations: Projections of the global $\Delta \chi^{2}$ functions onto each of the $\left(\delta m^{2}, \sin ^{2} \theta_{12}, \sin ^{2} \theta_{13}\right)$ parameters. The $n$-sigma bounds on each parameter (the others being unconstrained) correspond to $\Delta \chi^{2}=n^{2}$. The solid curves show the results of our previous analysis (see Fig. 9 in [1]). The dotted curves show the effect of the $\Delta m^{2}$ estimate in Eq. (31), which includes the revised SK atmospheric neutrino results from [12]. No significant change is seen in the fit to $\delta m^{2}$ and $\sin ^{2} \theta_{12}$. Conversely, the fit to the $\sin ^{2} \theta_{13}$ parameter becomes less constraining, the $3 \sigma$ upper bound being increased from 0.05 to 0.067 . 